\title{
Early BCR-ABL1 decline in imatinib-treated patients with chronic myeloid leukemia: results from a multicenter study of the Chinese CML alliance
}

\author{
Jingru Zhang ${ }^{1}$, Yingqiao Wang ${ }^{1}$, Jianxiang Wang ${ }^{2}$, Jianda $\mathrm{Hu}^{3}$, Suning Chen ${ }^{4}$, Jie $\mathrm{Jin}^{5}$, Ting Liu', Jianfeng Zhou',
} $\mathrm{Yu} \mathrm{Hu} \mathbf{u}^{8}$, Daoxin $\mathrm{Ma}^{1}$, Xiaojun Huang $\mathbb{1}^{9}$, Chunyan $\mathrm{Ji}^{1}$ and Ming $\mathrm{Hou}^{1}$

\begin{abstract}
An early molecular response is spectacularly predictive of outcome in chronic myeloid leukemia (CML) and early response landmarks may identify the high-risk patients likely to be benefit from an early therapy switch. In this study, we evaluated the most relevant cutoffs for early molecular response markers (BCR-ABL1 values at 3 months, log reduction and halving time between diagnosis and 3 months) in 476 first-line imatinib-treated Chinese patients with chronic phase CML. All outcomes were significantly superior for the 324 patients with 3 -month BCR-ABL1 $\leq 10 \%$, so did for the 270 patients with BCR-ABL1 $>0.61$ log reduction. BCR-ABL1 halving time $\leq 22$ days was identified for patients with the most favorable outcome. Moreover, the prognosis was significantly poorest for patients with both halving time $>44$ days and BCR-ABL1 $>10 \%$. Importantly, multivariate regression analysis demonstrated that a BCR-ABL1 log reduction calculated at 3 months of 0.61 was the only variable that significantly predicted for OS. Our results highlight the importance of rapid initial decline of BCR-ABL1 in predicting satisfactory outcome. Our data support the evidence that monitoring BCR-ABL1 values at an early time point could contribute to accurately assess response and ultimately guide clinical decisions regarding the timing of therapeutic intervention.
\end{abstract}

\section{Introduction}

Prognosis of patients with chronic myeloid leukemia (CML) has been dramatically improved with the introduction of imatinib as the first tyrosine kinase inhibitor $(\mathrm{TKI})^{1}$. Early molecular response (EMR) to TKI treatment has a strong predictive value in CML patients and early response landmarks may identify patients at higher risk for transformation and poor outcome, who would benefit from an early switch to second-line therapy ${ }^{2-4}$. Assessment of BCR-ABL1 at 3 months has been demonstrated

Correspondence: Daoxin Ma (daoxinma@hotmail.com) or

Xiaojun Huang (huangxiaojun@bjmu.edu.cn)

'Department of Hematology, Qilu Hospital, Shandong University, Jinan, Shandong, China

${ }^{2}$ Institute of Hematology and Blood Diseases Hospital, Chinese Academy of Medical Sciences and Peking Union Medical College, Tianjin, China

Full list of author information is available at the end of the article. to be the only indicator for predicting prognosis ${ }^{5}$. Indeed, BCR-ABL1 transcript level $\leq 10 \%$ on the international reporting scale (IS) at 3 months is consistently associated with significantly superior overall survival (OS), progression-free survival (PFS), event-free survival (EFS), failure-free survival (FFS), as well as cytogenetic and molecular responses ${ }^{6,7}$. Therefore, this molecular milestone value was incorporated into the National Comprehensive Cancer Network (NCCN) and European Leukemia Net (ELN) recommendations for the management of $\mathrm{CML}^{8}$.

Although EMR evaluation criteria seem promising, various factors such as baseline biological characteristics, treatment intensity and tolerance may have an impact on the treatment response and survival of CML patients 9 . Specifically, BCR-ABL1 transcript values at diagnosis are 
markedly different among chronic phase (CP) CML patients ${ }^{10,11}$. Since imatinib-treated newly diagnosed CML patients often require transient discontinuation of treatment during the early phase due to adverse events, treatment responses and outcomes may be underestimated by BCR-ABL1 transcript levels at 3 months ${ }^{2,3}$. A more precise prognosis evaluation method is urgently needed, which reflects individual clinical entities in patients with CML-CP.

Compared to the effect on the worldwide population

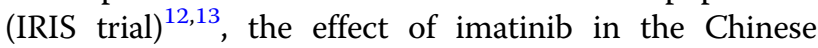
subset was relatively favorable while the incidence of nonhematological toxicities seemed to be more frequent, in part because Chinese patients have higher plasma concentrations of imatinib ${ }^{14,15}$. Recent studies proposed that the rate of BCR-ABL1 decline is a critical prognostic predictor in CML treated with TKI and patients at risk of disease progression can be identified precisely by the lack of a half-log reduction of BCR-ABL1 transcripts at 3 months $^{10,11}$. However, there is no evident information predicting treatment response and prognosis especially in the Chinese population, with the distinct clinical profile of TKI therapy.

In this multicenter study, we investigated the prognostic significance of patient baseline characteristics and EMR markers, as well as evaluated the ability of BCR-ABL1 kinetics to predict outcome in our cohort of 476 first-line imatinib-treated CML patients. Additionally, we explored a better early discriminator of the highest-risk/lowest-risk patients at 3 months, which would help to refine recommendations for treatment decisions at early time points.

\section{Materials and methods Patient sample}

From April 2000 to May 2016, a total of 693 patients with newly diagnosed CML from nine Chinese hospitals were enrolled in our clinical trials, data entry was closed on 23 November 2016. These trials included a subset of patients from Peking University People's Hospital $(n=197)$, Tianjin Institute of Hematology and Blood Diseases Hospital $(n=96)$, Fujian Medical University Union Hospital $(n=96)$, The First Affiliated Hospital of Soochow University $(n=80)$, The First Affiliated Hospital of Zhejiang University $(n=73)$, Qilu Hospital of Shandong University $(n=70)$, West China Hospital of Sichuan University $(n=44)$, Tongji Hospital of Huazhong University of Science and Technology $(n=23)$, and Union Hospital of Huazhong University of Science and Technology $(n=14)$. Only CML-CP patients who were $\geq 14$ years old and expressing typical BCR-ABL1 transcripts (b2a2, b3a2, or b2a2 and b3a2) were included. Besides, patients with imatinib onset before baseline sample collection as well as patients pretreated with hydroxyurea of more than 6 months were excluded from the analysis. A total of 476 imatinib-treated patients were investigated. BCR-ABL1 transcript levels at diagnosis and 3 months were available in 412 patients for review and were thereafter included in this study for final analysis. Written informed consent was obtained from each patient. This study was approved by the ethics committee at each participating institution and was conducted in accordance with the Declaration of Helsinki.

\section{Molecular analysis}

The expression levels of BCR-ABL1 transcripts were determined by quantitative real-time PCR as described previously ${ }^{16}$. ABL1 as the control gene has been studied extensively for suitability for BCR-ABL1 measurement ${ }^{5}$. Results were presented as $B C R-A B L 1 / A B L 1 \%^{\mathrm{IS}}$, with each participating laboratory-specific values converted to IS. Molecular monitoring was performed prior to commencing imatinib (baseline), at 3, 6, and 12 months, and every 3-6 months thereafter.

\section{Assessment of treatment response}

Cytogenetic and molecular responses were defined according to European Leukemia Net criteria ${ }^{8}$. Cytogenetic assessment was performed at baseline, 3, 6, and 12 months, and every 12 months after the achievement of complete cytogenetic remission (CCyR). If the optimal response has not been achieved, cytogenetic monitoring should be conducted every 3-6 months. The achievement of a major molecular response (MMR $\left.\leq 0.10 \%{ }^{\mathrm{IS}}\right)$ and molecular response $4.5\left(\mathrm{MR}^{4.5} \leq 0.0032 \%{ }^{\mathrm{IS}}\right)$ required confirmation at two consecutive measurements. OS was defined by absence of death from any reason, PFS by absence of accelerated phase (AP), blast crisis (BC) and death from any reason. EFS was measured from the start of treatment to the date of any of the following events while on therapy: loss of complete hematologic remission (CHR), loss of major cytogenetic remission (MCyR), progression to AP/BC or death from any cause ${ }^{17}$. Because of the limitations of this definition, we also measured FFS that accounts for other events such as lack of milestone responses at 3, 6, and 12 months, loss of cytogenetic or molecular response, acquisition of BCR-ABL1 mutations, clonal chromosomal abnormalities in $\mathrm{Ph}^{+}$cells, intolerance, or treatment discontinuation for any reason ${ }^{11}$.

\section{Log reduction of $B C R-A B L 1$ transcript and halving time calculation}

The rate of $\mathrm{BCR}-\mathrm{ABL} 1$ decline from baseline was assessed by estimating the log reduction and halving time of BCR-ABL1 values. The log reduction in BCR-ABL1 transcript level was defined as log (transcript level at diagnosis/transcript level at 3 months). The halving time defines the number of days over which the BCR-ABL1 transcripts achieve one-half of the baseline value, and was 
calculated as $\ln 2 \times c /[\ln (a)-\ln (b)]$ where (a) is the transcript value at diagnosis, (b) the transcript value of the 3month follow-up and (c) the number of days between both measurements. Where there was no BCR-ABL1 decline from baseline at 3 months, halving times were negative value ( $n=41$ in our study). To enable assessment of the discriminatory power of halving time, the halving times of those patients were imputed to the longest positive halving time of 8000 days, which was calculated for the patient with the smallest reduction ${ }^{11}$.

\section{Statistical analysis}

Receiver operating characteristic (ROC) curves were generated and area under the curve (AUC) was used to compare the correlations between factors and prognosis. The optimal thresholds along the ROC curves were determined using the Youden index. Survival probabilities were performed using the Kaplan-Meier method and compared by the log-rank test. Hazard ratios (HR) and 95\% confidential interval (CI) were derived using the Cox proportional hazard model. Cumulative incidence probability curves were calculated to analyze treatment response considering all permanent discontinuations of TKI for any reason as competing risks. Relative risks (RR) were calculated from Fine-Gray regression models, and significance was determined with the Wald test. Level of significance was 0.05 . All calculations were performed with the SPSS software Version 13.0.

\section{Results}

\section{Patient characteristics and outcomes}

Patient demographics and disease characteristics at the time of imatinib therapy are presented in Table 1. A total of 476 patients with a median age of 39 years were analyzed. The median follow-up duration of imatinib therapy was 34 months (range 2-126). Disease progression was observed in 24 patients (5.0\%), 16 of them died (3.4\%).

With regard to prognostic significance, survival analysis showed that age and gender had no detectable effect on clinical outcome and achievement of molecular response (Table 2). The mean daily doses (MDDs) of imatinib led to differences in the percentage of patients with $\leq 10 \%$ BCR-ABL1 at 3 months. Of the 348 evaluable patients with $\geq$ the median MDDs of $400 \mathrm{mg}, 249$ (71.6\%) had BCR-ABL1 values $\leq 10 \%$ at 3 months. In contrast, 74 of 125 (59.2\%) of patients treated with $<400 \mathrm{mg}$ had BCRABL1 values $\leq 10 \%$ at 3 months. However, no MDDs cutoff could be identified that allowed a discrimination concerning OS, PFS, EFS, or PFS, as well as no statistical difference in outcome was found among the dose groups (Table 2). Therefore, treatment dose was not considered as a prognostic factor for further analysis.
Table 1 Patient characteristics $(n=476)$

\begin{tabular}{|c|c|}
\hline & $N(\%)$ \\
\hline Age & 39 years (range $14-87$ ) \\
\hline Sex, male, $N(\%)$ & $301(63 \%)$ \\
\hline Sokal risk, N (\%) Low/INT/High/NA & $156(33) / 193(41) / 87(18) / 40(8)$ \\
\hline $\begin{array}{l}\text { Interval since diagnosis, months, median } \\
\text { (range) }\end{array}$ & $0.3(0-6)$ \\
\hline $\begin{array}{l}\text { MDDs of imatinib (mg/day), median } \\
\text { (range) }\end{array}$ & $400(112.6-748.7)$ \\
\hline \multicolumn{2}{|l|}{ MDDs of imatinib (mg/day), $n$ (\%) } \\
\hline$\geq 100$ to $<400$ & $125(26 \%)$ \\
\hline 400 & $304(64 \%)$ \\
\hline$>400$ to $\leq 800$ & $47(10 \%)$ \\
\hline $\begin{array}{l}\text { Baseline } B C R-A B L 7^{15} \text { transcript }(\%), \text { median } \\
\text { (range) }(N A=61)\end{array}$ & $43.20(0.0114-598.64)$ \\
\hline $\begin{array}{l}\text { 3-month } B C R-A B L 1^{1 S} \text { transcript }(\%) \text {, } \\
\text { median (range) }(N A=3)\end{array}$ & $3.55(0.0025-141.62)$ \\
\hline
\end{tabular}

NA not available, INT intermediate, MDDs mean daily doses

\section{Predictive impact of BCR-ABL1 transcript levels at diagnosis}

BCR-ABL1 transcript levels at diagnosis varied in a wide range $(0.0114-598.64 \%)$ with a median of $43.20 \%$. In order to determine whether the transcript level at diagnosis could be used as a prognostic indicator for disease progression, we compared patients who achieved $\leq 10 \%$ BCR-ABL1 at 3 months $(n=281)$ and those who did not $(n=131)$. The BCR-ABL1 median ratio at diagnosis was $42.80 \%$ in the first group and $44.77 \%$ in the second, and no significant difference was found between them. Besides, no prognostic cutoff could be identified for BCRABL1 transcript levels at diagnosis (Table 2).

\section{Prognostic significance of 3-month 10\% BCR-ABL1 cutoff}

Of the total 476 patients, 473 had a BCR-ABL1 assessment at 3 months and median values was $3.55 \%$ (range $0.0025-141.62 \%)$. It was recently demonstrated that the persistence of BCR-ABL1 transcript levels $>10 \%$ at 3 months identified a group of high-risk patients that would benefit from treatment optimization ${ }^{5,6}$. As expected, the 324 patients (68\% of all patients) with BCR-ABL1 values $\leq 10 \%$ at 3 months had significantly better outcome than those with $>10 \%$ ( $n=149,31 \%$ of all patients). The outcomes comparing $\leq 10 \%$ vs $>10 \%$ BCR-ABL1 were as follows: 3-year OS, $98.5 \%$ vs $94.0 \%, P=0.006$; 3 -year PFS, $97.8 \%$ vs $89.9 \%, P<0.001$; 3 -year EFS, $95.7 \%$ vs $87.2 \%$, $P=0.001$; 3 -year FFS, $81.5 \%$ vs $44.3 \%, P<0.001$; 1 -year CCyR, $81.5 \%$ vs $45.0 \%, P<0.001 ; 1.5$-year MMR, $67.0 \%$ vs 
Table 2 Probabilities of OS, PFS, EFS, and PFS at 3 years as well as CCyR at 1 years, MMR at 1.5 years and MR4.5 at 3 years by univariate analysis of baseline variables and the $B C R-A B L 1$ value, log reduction, halving time at 3 months

\begin{tabular}{|c|c|c|c|c|c|c|c|c|c|c|c|c|c|c|c|c|c|c|c|c|c|c|}
\hline Variable & No. & $\begin{array}{l}\text { OS } \\
\text { (\%) }\end{array}$ & $\begin{array}{l}\text { Hazard } \\
\text { ratio }\end{array}$ & $\begin{array}{l}P \\
\text { value }\end{array}$ & $\begin{array}{l}\text { PFS } \\
(\%)\end{array}$ & $\begin{array}{l}\text { Hazard } \\
\text { ratio }\end{array}$ & $\begin{array}{l}P \\
\text { value }\end{array}$ & $\begin{array}{l}\text { EFS } \\
(\%)\end{array}$ & $\begin{array}{l}\text { Hazard } \\
\text { ratio }\end{array}$ & $\begin{array}{l}P \\
\text { value }\end{array}$ & $\begin{array}{l}\text { FFS } \\
\text { (\%) }\end{array}$ & $\begin{array}{l}\text { Hazard } \\
\text { ratio }\end{array}$ & $\begin{array}{l}P \\
\text { value }\end{array}$ & $\begin{array}{l}\text { CCyR } \\
(\%)\end{array}$ & $\begin{array}{l}\text { Relative } \\
\text { risk }\end{array}$ & $\begin{array}{l}P \\
\text { value }\end{array}$ & $\begin{array}{l}\text { MMR } \\
(\%)\end{array}$ & $\begin{array}{l}\text { Relative } \\
\text { risk }\end{array}$ & $\begin{array}{l}P \\
\text { value }\end{array}$ & $\begin{array}{l}\mathrm{MR}^{4.5} \\
(\%)\end{array}$ & $\begin{array}{l}\text { Relative } \\
\text { risk }\end{array}$ & $\begin{array}{l}P \\
\text { value }\end{array}$ \\
\hline \multicolumn{23}{|c|}{ Age (median years) } \\
\hline$\leq 39$ & 215 & 97.7 & 1 & 0.239 & 94.9 & 1 & 0.847 & 92.1 & 1 & 0.991 & 66.5 & 1 & 0.575 & 73.0 & 1 & 0.146 & 56.3 & 1 & 0.136 & 24.2 & 1 & 0.594 \\
\hline$>39$ & 200 & 95.5 & 1.927 & & 94.5 & 1.071 & & 92.0 & 1.004 & & 69.5 & 0.907 & & 66.5 & 0.842 & & 50.0 & 0.817 & & 27.0 & 1.109 & \\
\hline \multicolumn{23}{|l|}{ Sex, $n(\%)$} \\
\hline Female & 157 & 98.7 & 1 & 0.083 & 94.9 & 1 & 0.839 & 92.4 & 1 & 0.766 & 70.7 & 1 & 0.21 & 67.5 & 1 & 0.320 & 55.4 & 1 & 0.961 & 27.4 & 1 & 0.989 \\
\hline Male & 258 & 95.3 & 3.752 & & 94.6 & 1.094 & & 91.9 & 1.114 & & 66.3 & 1.257 & & 71.3 & 1.129 & & 51.9 & 0.993 & & 24.4 & 0.997 & \\
\hline \multicolumn{23}{|c|}{ MDDs (mg/day), $n(\%)$} \\
\hline $\begin{array}{l}\geq 100 \text { to } \\
<400\end{array}$ & 117 & 95.7 & 1 & 0.721 & 93.2 & 1 & 0.611 & 90.6 & 1 & 0.802 & 55.6 & 1 & 0.015 & 61.5 & 1 & 0.155 & 52.1 & 1 & 0.096 & 27.4 & 1 & 0.272 \\
\hline 400 & 258 & 97.3 & 0.844 & & 95.7 & 0.839 & & 93.0 & 0.849 & & 74.0 & 0.739 & & 74.0 & 1.073 & & 55.0 & 0.986 & & 25.2 & 0.99 & \\
\hline $\begin{aligned} & >400 \text { to } \\
\leq & 800\end{aligned}$ & 40 & 95.0 & 1.051 & & 92.5 & 1.036 & & 90.0 & 1.033 & & 65.0 & 0.810 & & 67.5 & 1.463 & & 45.0 & 1.391 & & 22.5 & 1.287 & \\
\hline \multicolumn{23}{|c|}{ Baseline $B C R / A B L 1^{15}$ transcript } \\
\hline$\leq 43 \%$ & 207 & 95.7 & 1 & 0.341 & 93.2 & 1 & 0.261 & 89.9 & 1 & 0.160 & 63.3 & 1 & 0.123 & 74.4 & 1 & 0.628 & 55.1 & 1 & 0.252 & 27.1 & 1 & 0.994 \\
\hline$>43 \%$ & 208 & 97.6 & 0.588 & & 96.2 & 0.608 & & 94.2 & 0.601 & & 72.8 & 0.763 & & 65.4 & 0.767 & & 51.4 & 0.857 & & 24.0 & 1.001 & \\
\hline \multicolumn{23}{|c|}{ 3-month $B C R / A B L 1^{15}$ transcript } \\
\hline$\leq 10 \%$ & 281 & 98.2 & 1 & 0.012 & 97.5 & 1 & 0.001 & 95.0 & 1 & 0.001 & 80.1 & 1 & 0.001 & 81.5 & 1 & 0.001 & 66.5 & 1 & 0.001 & 35.6 & 1 & 0.001 \\
\hline$>10 \%$ & 131 & 93.1 & 4.045 & & 88.5 & 4.881 & & 85.5 & 3.104 & & 42.7 & 4.089 & & 45.0 & 0.354 & & 24.4 & 0.269 & & 4.6 & 0.134 & \\
\hline \multicolumn{23}{|c|}{ Log reduction } \\
\hline$>0.61$ & 270 & 98.5 & 1 & 0.007 & 97.8 & 1 & 0.001 & 95.6 & 1 & 0.001 & 82.6 & 1 & 0.001 & 79.3 & 1 & 0.001 & 66.3 & 1 & 0.001 & 34.8 & 1 & 0.001 \\
\hline$\leq 0.61$ & 142 & 93.0 & 4.894 & & 88.7 & 5.245 & & 85.2 & 3.460 & & 40.8 & 4.720 & & 52.1 & 0.475 & & 28.2 & 0.347 & & 8.5 & 0.270 & \\
\hline \multicolumn{23}{|l|}{ Halving time } \\
\hline$\leq 22$ days & 166 & 98.8 & 1 & 0.036 & 98.2 & 1 & 0.016 & 97.0 & 1 & 0.004 & 84.9 & 1 & 0.001 & 86.1 & 1 & 0.001 & 78.3 & 1 & 0.001 & 45.2 & 1 & 0.001 \\
\hline$>22$ days & 246 & 95.1 & 4.203 & & 92.3 & 4.458 & & 88.6 & 4.010 & & 56.9 & 3.646 & & 64.2 & 0.435 & & 36.2 & 0.323 & & 12.6 & 0.260 & \\
\hline
\end{tabular}

OS overall survival, PFS progression-free survival, EFS event-free survival, FFS failure-free survival, CCyR complete cytogenetic remission, MMR major molecular response, $M R^{4.5}$ molecular response $4.5, M D D$ s mean daily doses

24.8\%, $P<0.001$; and 3 -year $\mathrm{MR}^{4.5}, 34.6 \%$ vs $4.7 \%, P<$ 0.001 (Fig. 1).

Notwithstanding the usefulness of 3-month $10 \%$ BCRABL1 values for outcome prediction, there was a considerable number of patients with BCR-ABL1 $>10 \%$ who did not fail to therapy and some subsequently achieve satisfactory outcomes despite being initially categorized as a poor responder at 3 months (Table 3). Sixty-three of 149 patients $(42.3 \%)$ with $>10 \%$ BCR-ABL1 achieved an MMR, which was maintained in 60 patients $(95.2 \%)$ at the recent follow-up (median 18 months; range, 3-98.9 months). Furthermore, those patients with BCR-ABL1 $\leq 10 \%$ were classified as a good responder at 3 months; however, not all patients within this subgroup reached an optimal response and eventually favorable outcome (Table 3 ). At the first 18-month follow-up, 107 of 324 patients with $\leq 10 \%$ BCR-ABL1 had not achieved an MMR, which was sustained in 72 patients $(67.3 \%)$ during the succeeding 18 months follow-up. Of those patients with $\leq 10 \%$ at 3 months, 2 were already dead and 5 subsequently progressed, as well as 11 lost CHR and MCyR, which occurred before 24 months. Thus, 3-month 10\% BCRABL1 cutoff could not fully predict treatment response and ultimately clinical outcome, which urgently needs to seek the novel indicators for more precise and individual prognosis evaluation.
BCR-ABL1 log reduction between diagnosis and 3 months

We thereafter investigated whether another cutoff point could predict long-term achievement of molecular response and outcome in our cohort. Based on the fact that the initial tumor burden might reflect different extents of an ongoing disease $\mathrm{e}^{18,19}$, our study focused on the kinetics of the BCR-ABL1 decline from the individual patient baseline point to the 3-month values. We observed that some patients had very little or no decline, whereas others had more than a 50-fold reduction. Moreover, patients with the same BCR-ABL1 values at 3 months had better outcomes if their baseline values were higher. These findings demonstrated that the velocity of BCR-ABL1 decline may be critical for prognosis.

We therefore estimated the log reduction of 3-month BCR-ABL1 values from the individual baseline values and found that lower $\log$ reduction indicated a slow or no decline of BCR-ABL1, suggesting unsatisfactory outcomes. Of 412 evaluable patients, the median reduction was a 10.23-time decrease, corresponding to a $1.01 \mathrm{log}$ reduction. Using ROC analysis, the optimal log reduction thresholds for discriminating between outcomes were as follows: OS, 0.61 (AUC, 0.727; 95\% CI, 0.582-0.872); PFS, 0.61 (AUC, 0.734; 95\% CI, 0.628-0.841); EFS, 0.71 (AUC, 0.713; 95\% CI, 0.626-0.801); FFS, 0.91 (AUC, 0.745; 95\% CI, 0.693-0.798); CCyR, 0.68 (AUC, 0.782; 95\% CI, 

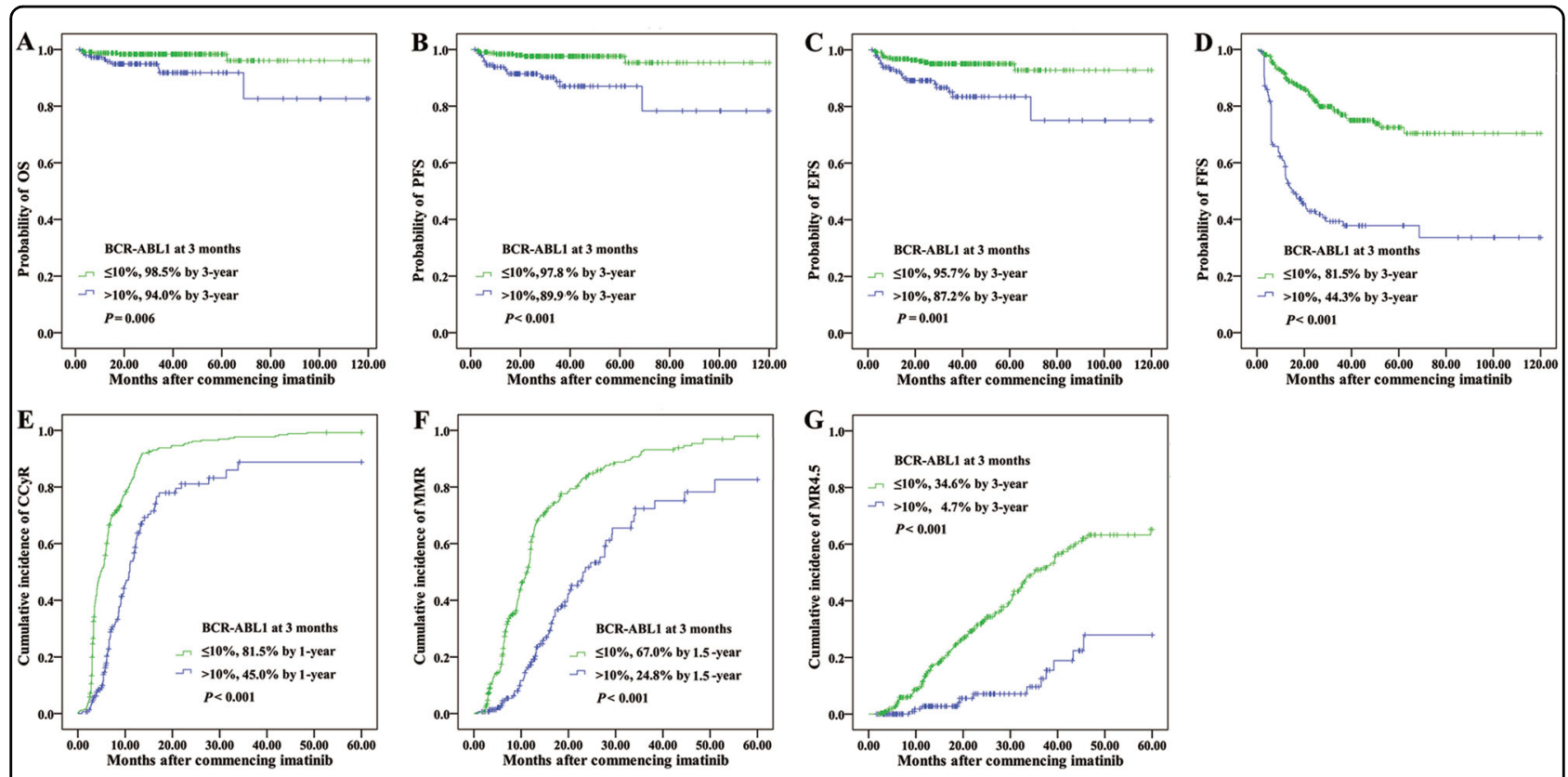

Fig. 1 Outcomes according to 3-month BCR-ABL1 value. a OS, b PFS, c EFS, d FFS, e CCYR, $\mathbf{f} M M R$, and $\mathbf{g} M R^{4.5}$

0.729-0.836); MMR, 0.91 (AUC, 0.759; 95\% CI, $0.711-0.807$ ); and $\mathrm{MR}^{4.5}, 1.18$ (AUC, 0.742; 95\% CI, $0.693-0.792)$. Of the highly relevant outcomes OS and PFS, we identified the optimal value of $0.61 \mathrm{log}$ reduction at 3 months as the best predictive cutoff for outcome evaluation.

Those patients with BCR-ABL1 >0.61 log reduction (270/412 evaluable patients, 66\%) had significantly superior outcomes compared with the 142 of 412 patients (34\%) where the log reduction was $\leq 0.61$ (3-year OS, 98.5\% vs $93.0 \%, P=0.003$; 3 -year PFS, $97.8 \%$ vs $88.7 \%, P<$ 0.001 ; 3-year EFS, $95.6 \%$ vs $85.2 \%, P<0.001$; 3 -year FFS, $82.6 \%$ vs $40.8 \%, P<0.001$; 1 -year CCyR, $79.3 \%$ vs $52.1 \%, P$ $<0.001$; 1.5 -year MMR, $66.3 \%$ vs $28.2 \%, P<0.001$; and $3-$ year MR4.5, $34.8 \%$ vs $8.5 \%, P<0.001$, Fig. 2). Similarly with 3 -month $10 \%$ BCR-ABL1, the $0.61 \log$ reduction cutoff in our study was demonstrated to be another effective predictor of survival (Table 2).

Of note, we also evaluated the discriminatory power for outcome prediction of the $0.61 \log$ reduction combined with $10 \%$ cutoff at 3 months. Four groups were defined as follows: group I included those who achieved BCR-ABL1 transcript levels $\leq 10 \%$ and $>0.61 \log$ reduction both at 3 months. Groups II included those who achieved BCRABL1 transcript levels $\leq 10 \%$ and $\leq 0.61$ log reduction both at 3 months. Groups III included those who failed to achieve an EMR (BCR-ABL1 >10\%) and >0.61 log reduction both at 3 months. Groups IV included those who failed to achieve an EMR and $\leq 0.61 \log$ reduction both at 3 months. When outcomes were compared among these four groups, statistical differences were noted in OS,
PFS, EFS, and FFS at 3 years as well as CCyR at 1 year, MMR at 1.5 years and $M^{4.5}$ at 3 years (Fig. 3 and Supplementary Table 1). Consistently, subgroup analyses comparing Group I vs IV showed differences in OS $(98.4 \%$ vs $91.8 \%, P=0.005)$, PFS (98.0\% vs $87.3 \%, P<0.001)$, EFS (95.6\% vs $83.6 \%, P<0.001)$, FFS $(83.1 \%$ vs $36.4 \%, P<$ $0.001)$, CCyR (81.1\% vs $42.7 \%, P<0.001)$, MMR (69.1\% vs $22.7 \%, P<0.001)$ and $\mathrm{MR}^{4.5}(37.3 \%$ vs $4.5 \%, P<0.001)$ in favor of Group I. Comparing Groups I and II, Group II showed inferior FFS (83.1\% vs $56.3 \%$ at 3 years, $P=0.001)$, but no difference in OS $(98.4 \%$ vs $96.9 \%, P=0.557)$, PFS (98.0\% vs $96.9 \%, P=0.181)$ and EFS (95.6\% vs $90.6 \%$, $P=0.026)$. However, when subgroup analyses were restricted to Groups I and III, there were no differences in OS $(98.4 \%$ vs $100 \%, P=0.706)$, PFS $(98.0 \%$ vs $95.2 \%$, $P=0.405)$, EFS $(95.6 \%$ vs $95.2 \%, P=0.885)$ and FFS (83.1\% vs $76.2 \%, P=0.338$ ) between those two groups. Together, the 110 patients (26.7\%) with BCR-ABL1 >10\% and $\leq 0.61 \log$ reduction at 3 months were identified to have the poorest survival.

Importantly, multivariate regression analysis demonstrated that a BCR-ABL1 log reduction calculated at 3 months of 0.61 was the only variable that significantly predicted for OS (HR, 6.539; 95\% CI, 1.512-28.283; $P=$ 0.012).

\section{BCR-ABL1 halving time}

We investigated whether the patient with the most favorable outcome could be identified at an earlier time point. $B C R-A B L 1$ halving time was examined for this purpose. As illustrated in Supplementary Fig. 1, BCR- 


\begin{tabular}{|c|c|c|c|c|c|c|}
\hline & BCR-ABL1 $1^{\text {IS }}$ & $\mathrm{N}$ & $(\%)$ & BCR-ABL1 $^{\text {IS }}$ & $\mathrm{N}$ & $(\%)$ \\
\hline 3-month & $>10 \%$ & 149 & & $\leq 10 \%$ & 324 & \\
\hline \multirow[t]{5}{*}{ 6-month } & $>10 \%$ & 35 & $23.49 \%$ & $>10 \%$ & 6 & $1.85 \%$ \\
\hline & $>1 \%-10 \%$ & 59 & $39.60 \%$ & $>1 \%-10 \%$ & 44 & $13.58 \%$ \\
\hline & $>0.1 \%-1 \%$ & 27 & $18.12 \%$ & $>0.1 \%-1 \%$ & 131 & $40.43 \%$ \\
\hline & $\leq 0.1 \%$ & 5 & $3.36 \%$ & $\leq 0.1 \%$ & 107 & $33.02 \%$ \\
\hline & NA & 23 & $15.44 \%$ & NA & 36 & $11.11 \%$ \\
\hline \multirow[t]{5}{*}{ 12-month } & $>10 \%$ & 14 & $9.40 \%$ & $>10 \%$ & 1 & $0.31 \%$ \\
\hline & $>1 \%-10 \%$ & 32 & $21.48 \%$ & $>1 \%-10 \%$ & 17 & $5.25 \%$ \\
\hline & $>0.1 \%-1 \%$ & 31 & $20.81 \%$ & $>0.1 \%-1 \%$ & 56 & $17.28 \%$ \\
\hline & $\leq 0.1 \%$ & 32 & $21.48 \%$ & $\leq 0.1 \%$ & 175 & $54.01 \%$ \\
\hline & NA & 40 & $26.85 \%$ & NA & 75 & $23.15 \%$ \\
\hline
\end{tabular}

Green, yellow, and red color represent optimal, warning, and failure, respectively.

Table 3 Distribution of $B C R-A B L 1$ values at 3, 6, and 12 months with $0.1 \%$ IS, $1 \%$ IS, and $10 \%$ IS as cutoff values

ABL1 values decline from baseline is exponential, demonstrating the halving time calculation is valid. We performed ROC analysis to determine the optimal halving time thresholds to predict outcome: OS, 44 days (AUC, 0.731; 95\% CI, 0.585-0.877); PFS, 44 days (AUC, 0.736; 95\% CI, 0.629-0.842); EFS, 39 days (AUC, 0.715; 95\% CI, 0.627-0.803); FFS, 30 days (AUC, 0.744; 95\% CI, 0.691-0.797); MMR, 28 days (AUC, 0.766; 95\% CI, 0.721-0.811); and $\mathrm{MR}^{4.5}$, 22 days (AUC, 0.760 ; $95 \% \mathrm{CI}$, $0.710-0.809$ ). Since this predictive marker could contribute to assess early response to first-line TKI and relieve the potentially psychological burden of patients, we selected the optimal $\mathrm{MR}^{4.5}$ halving time of 22 days for further outcome prediction in favor of sensitivity.

Of the 166 patients (40.3\%) with BCR-ABL1 halving time $\leq 22$ days, only one patients did not achieved $\leq 10 \%$ BCR-ABL1 at 3 months, whereas was still survival without progression during 5-year follow-up. Patients with halving time $\leq 22$ days had significantly superior outcomes compared with patients whose BCR-ABL1 values did not halve by 22 days $(n=246$; 3 -year OS, $98.8 \%$ vs $95.1 \%, P=0.041$; 3-year PFS, $98.2 \%$ vs $92.3 \%, P=0.008$; 3-year EFS, $97.0 \%$ vs $88.6 \%, P=0.002$; 3 -year FFS, $84.9 \%$ vs $56.9 \%, P<0.001$; and 1 -year CCyR, $86.1 \%$ vs $64.2 \%, P=0.017$; 1.5 -year MMR, $78.3 \%$ vs $36.2 \%, P<0.001$; 3 -year $\mathrm{MR}^{4.5}, 45.2 \%$ vs $12.6 \%, P=0.003$, Fig. 4 and Table 2). Therefore, BCRABL1 halving time $\leq 22$ days could be recognized as a critical prognostic discriminator for the best prognosis patients.

Our above study illustrated that among the patients with $>10 \%$ at 3 months, minimal or no decline from the baseline BCR-ABL1 values indicated the high risk of unfavorable outcome. In order to improve response and thereby minimize exposure to risk over time, we would like to find a better early indicator of the poorest-risk patients, who could benefit from alternative treatment as early as possible. Data showed that all outcomes were significantly inferior for the 142 patients (34.5\%) with halving time $>44$ days $(P<0.01$, equal to $\log$ reduction $\leq 0.61$ ). More importantly, among the $31.8 \%$ of evaluable patients in our cohort with BCR-ABL1 $>10 \%$ at 3 months, BCR-ABL1 halving time $>44$ days was statistically associated with the worst outcomes $(n=110$; 3 -year OS 91.8\%, PFS 87.3\%, EFS 83.6\%, FFS 36.4\% and $\mathrm{MR}^{4.5} 4.5 \%$; Group D in Supplementary Table 2), demonstrating that lack of a BCR-ABL1 decline or a slow decline from baseline conveyed the highest risk of treatment failure, progression, and death. The comparison of clinical outcome and response to imatinib therapy among the group A-D were presented in Fig. 5 with respect to halving time (22 and 44 days) and 3-month 10\% BCR-ABL1 values.

\section{Discussion}

In the present study, we confirmed the strong predictive value of three EMR: 3-month BCR-ABL1 values, log reduction, and halving time of BCR-ABL1 levels between diagnosis and 3 months. Our results suggested that patients with BCR-ABL1 values on a constant and rapid downward trajectory had a high chance of reaching an optimal response and ultimately achieving a favorable outcome. However, among the patients with $>10 \%$ at 3 months, lack of a BCR-ABL1 decline or a slow decline from baseline conveyed the highest risk of treatment failure, progression, and death.

High BCR-ABL transcript levels before treatment were reported to be associated with inferior probabilities of optimal response $\mathrm{e}^{18,20}$. In contrast, recent studies demonstrated that BCR-ABL values at diagnosis cannot predict the achievement of molecular response and patient survival $^{10,21}$. Consistent with the absence of prognostic value 

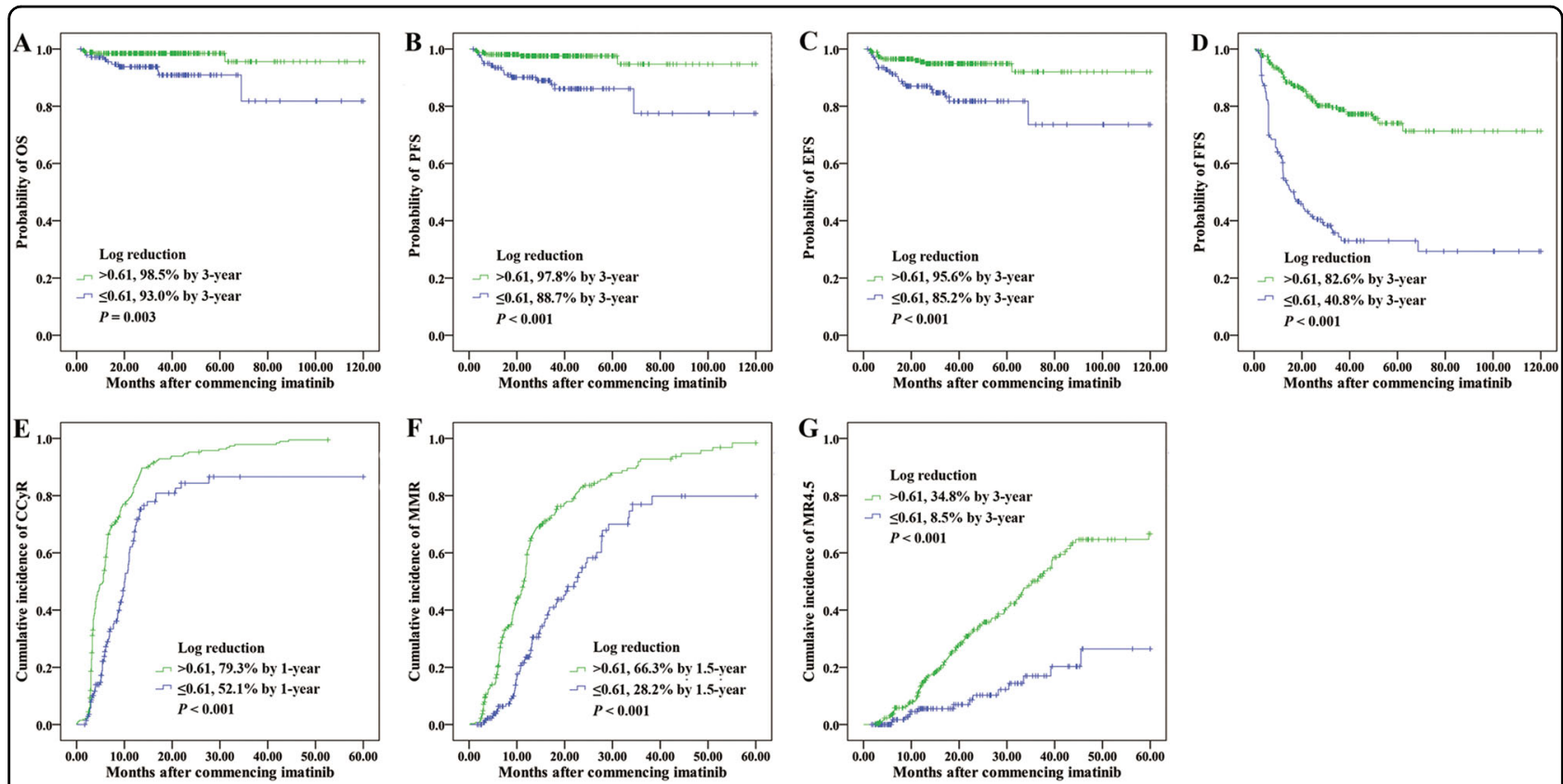

Fig. 2 Outcomes according to BCR-ABL1 log reduction. a OS, b PFS, c EFS, d FFS, e CCYR, f MMR, and $\mathbf{g} M \mathrm{R}^{4.5}$
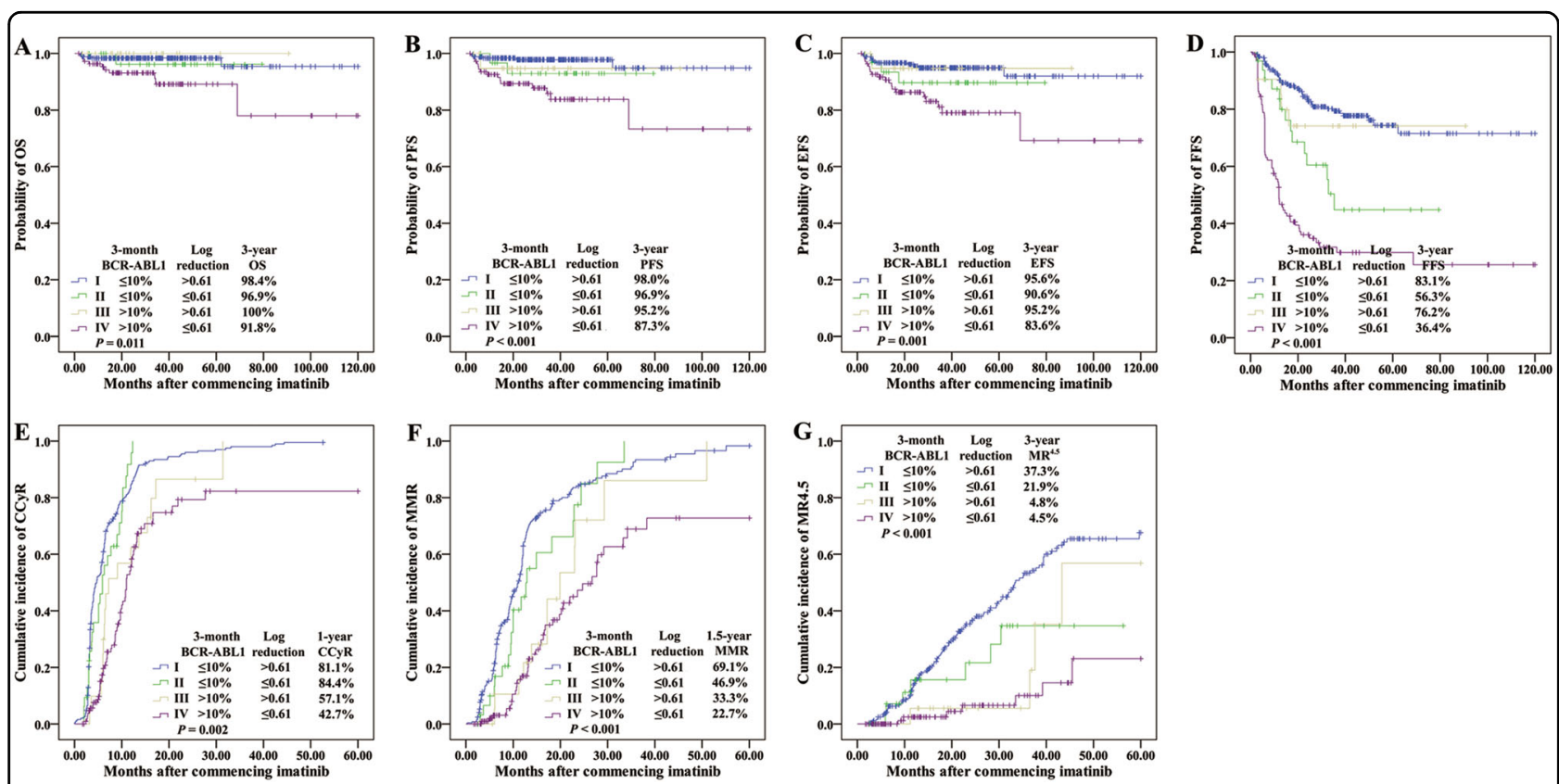

Fig. 3 Outcomes according to both 3-month BCR-ABL1 value and log reduction. a OS, b PFS, c EFS, d FFS, e CCYR, f MMR, and $\mathbf{g} M R^{4.5}$

of pre-treatment transcript levels, we did not found any predictive cutoff with regard to survival. This may be because the emergence of additional mutations was rare at diagnosis, and resulted from the outgrowth of the BCRABL1-positive clone, high BCR-ABL values were elevated as the disease progressed.
In the current era of various TKIs, the early selection of patients requiring alternative treatment is critical. Previous studies demonstrated the prognostic value of 3-month BCR-ABL1 transcript levels ${ }^{5,6}$. Marin et al. identified cutoffs in the 3-month transcript levels (9.84\%) that are predictors for OS using ROC curves ${ }^{5}$. Hanfstein et al. 

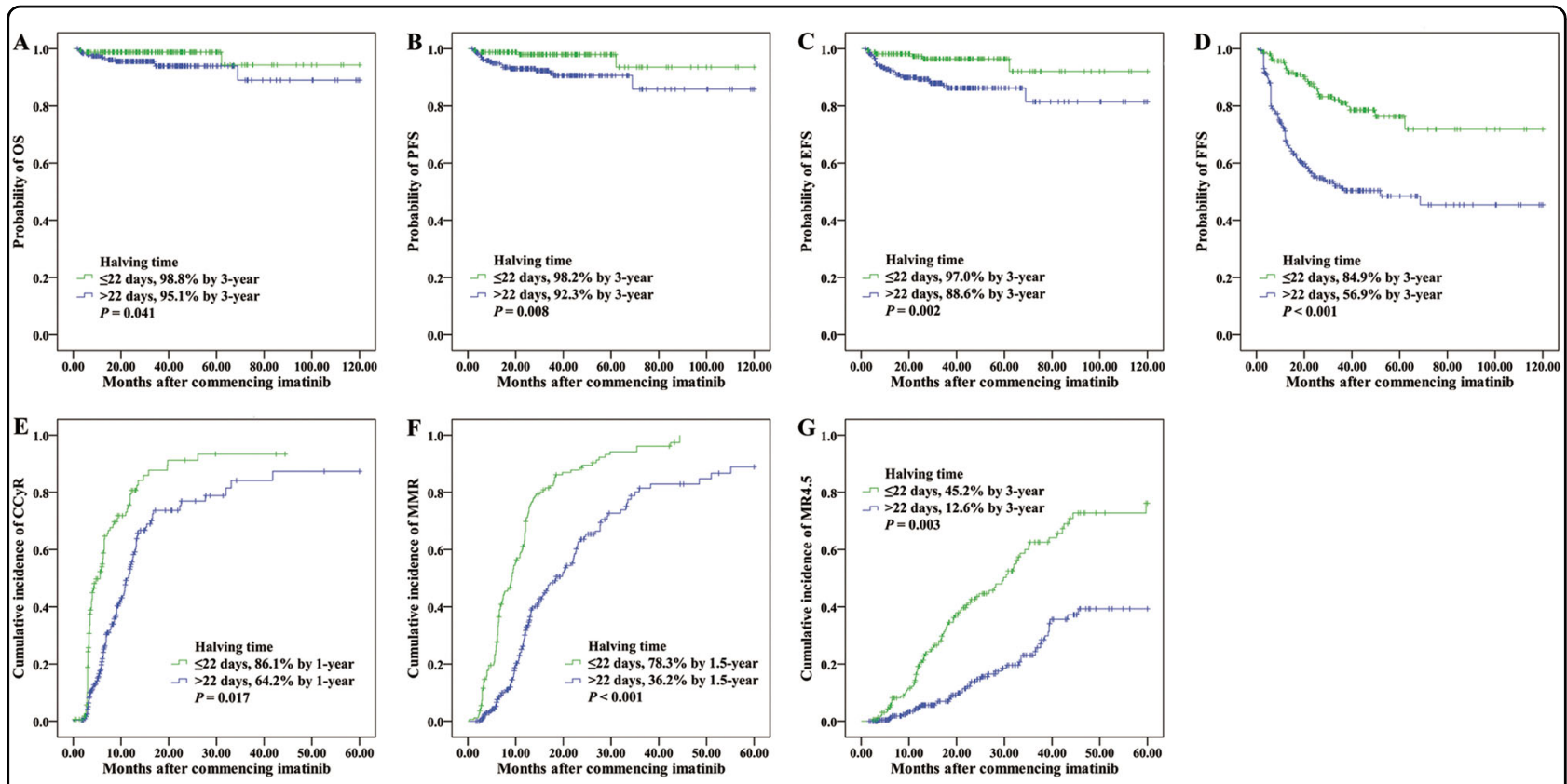

Fig. 4 Outcomes according to BCR-ABL1 halving time. a OS, b PFS, c EFS, d FFS, e CCYR, f MMR, and $\mathbf{g} M R^{4.5}$
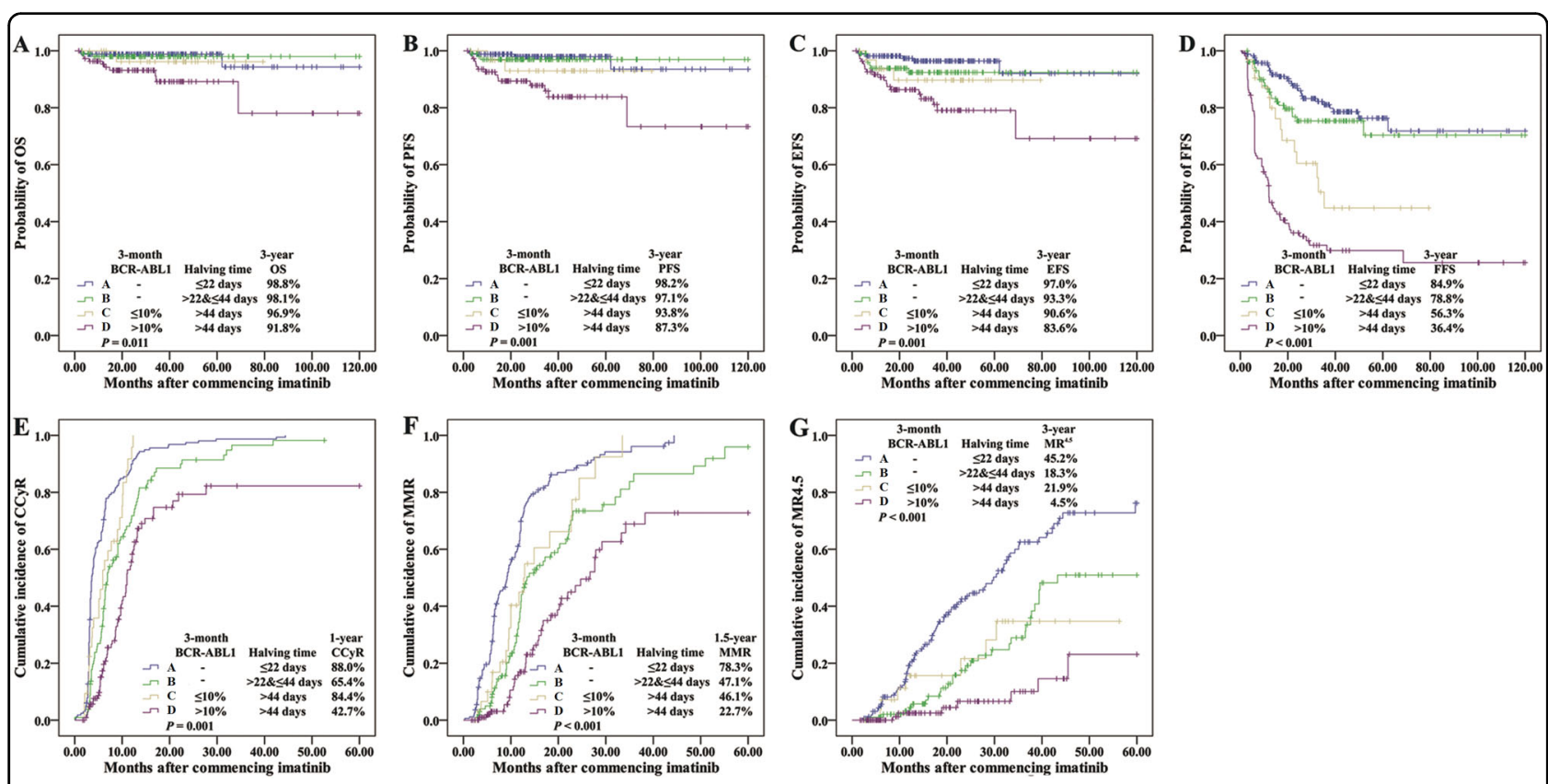

Fig. 5 Outcomes according to both 3-month BCR-ABL1 value and halving time. a OS, b PFS, c EFS, d FFS, e CCyR, f MMR, and $\mathbf{g} M R^{4.5}$

reported the predictive role of BCR-ABL1 transcript levels of $10 \%$ at 3 months for PFS and OS $^{6}$. Consistently, our analysis verified that 3-month EMR was also significantly associated with higher cytogenetic and molecular response rates. The estimation of 3-month EMR predicted higher survivals with a decreased risk of progression.

It is no surprising that the prediction based on the rate of reduction seems to be more precise than the use of isolated 3-month BCR-ABL1 values ${ }^{10,11,22}$. In our study, the molecular $0.61-\log$ reduction landmark (equal to 44 days-halving time) yields a relatively small $P$ value and high Hazard ratio when compared with 3-month $10 \%$ $B C R-A B L 1$ values, indicating a more precise prediction. Hanfstein et al. found that the lack of a $0.46-\log$ reduction of BCR-ABL1 transcripts at 3 months was a discriminator of patients at risk for disease progression ${ }^{10}$. Branford et al. 
emphasized that the rate of BCR-ABL1 decline as assessed by BCR-ABL1 halving time (76 days) was a critical predictor for very poor outcomes among patients with BCRABL1 $>10 \%$ at 3 months ${ }^{11}$. Thus, the velocity of early transcript elimination in the Chinese population accordingly appeared to be earlier than those in the west, possibly due to the relatively higher imatinib plasma levels in the Chinese patients ${ }^{14,15}$. Previous reports showed that imatinib trough level on day 29 were significantly associated with an achievement of 3-month EMR ${ }^{9,23}$.

Since prognostic information available even as early as 3 months may be too late for effective intervention in patients who experience early transformation ${ }^{24}$, we evaluated this optimal time point as being 22 days after the start of treatment in our cohort. Patients with a more rapid decline (BCR-ABL1 halving time $\leq 22$ days) had a higher chance of ultimately achieving MMR and therefore a better prognosis. Therefore, BCR-ABL1 halving time $\leq 22$ days was identified as a better early cutoff for patients with the most favorable outcome.

Additionally, our study highlights the importance of performing molecular analysis periodically to assess the BCR-ABL1 decline over the critical first 3 months, which may provide a cost-effective process for the better identification of patients for whom the risks and potential additional drug costs of therapy change are justified. Therefore, in a near future the survival prediction of each patient to TKI therapy will not only use the raw transcript levels but also different time-dependent variables assessing the BCR-ABL1 kinetics which are predictive of future molecular response and survival. Standardization of halving time or velocity of reduction will likely help establish more stringent recommendation and modify current clinical practices.

In conclusion, our study demonstrated that patients with CML-CP treated with imatinib can be stratified according to the BCR-ABL1 kinetics and that this stratification might contribute to the timing and necessity of therapeutic intervention. Because TKI therapy might have regional effects and might reflect distinct patient clinical profiles, we hope to validate these findings by studying larger cohorts in each region in the near future.

\section{Acknowledgements}

This work was supported by the grant from the Natural Science Foundation of China $(81400118,81470319,91642110,81370662$, and 91442126).

\section{Authors' contributions}

X.H. and D.M. designed the research study. J.W., J.H., S.C., J.J., T.L., J.Z., Y.H., D.M., X.H., C.J., and M.H. performed the research. J.Z. and Y.W. analyzed the data. J.Z. wrote the paper.

\section{Author details}

'Department of Hematology, Qilu Hospital, Shandong University, Jinan, Shandong, China. ${ }^{2}$ Institute of Hematology and Blood Diseases Hospital, Chinese Academy of Medical Sciences and Peking Union Medical College,
Tianjin, China. ${ }^{3}$ Fujian Provincial Key Laboratory of Hematology, Fujian Institute of Hematology, Fujian Medical University Union Hospital, Fuzhou, Fujian, China. ${ }^{4}$ Jiangsu Institute of Hematology, Key Laboratory of Thrombosis and Hemostasis of Ministry of Health, The First Affiliated Hospital of Soochow University, Suzhou, Jiangsu, China. ${ }^{5}$ Department of Hematology, The First Affiliated Hospital, Zhejiang University College of Medicine, Hangzhou, Zhejiang, China. ${ }^{6}$ Department of Hematology, West China Hospital, Sichuan University, Chengdu, Sichuan, China. ${ }^{7}$ Department of Hematology, Tongji Hospital, Tongji Medical College, Huazhong University of Science and Technology, Wuhan, Hubei, China. ${ }^{8}$ Institute of Hematology, Union Hospital, Tongji Medical College, Huazhong University of Science and Technology, Wuhan, Hubei, China. Institute of Hematology, Peking University People's Hospital, Beijing, China

\section{Conflict of interest}

The authors declare that they have no conflict of interest.

\section{Publisher's note}

Springer Nature remains neutral with regard to jurisdictional claims in published maps and institutional affiliations.

Supplementary Information accompanies this paper at https://doi.org/ 10.1038/s41408-018-0093-4.

Received: 20 January 2018 Revised: 16 April 2018 Accepted: 26 April 2018 Published online: 15 June 2018

\section{References}

1. Hehlmann, R., Hochhaus, A., Baccarani, M. \& European, L. Chronic myeloid leukaemia. Lancet 370, 342-350 (2007).

2. Jabbour, E. et al. Early response with dasatinib or imatinib in chronic myeloid leukemia: 3-year follow-up from a randomized phase 3 trial (DASISION). Blood 123, 494-500 (2014).

3. Fujisawa, S. et al. Efficacy and safety of dasatinib versus imatinib in Japanese patients with newly diagnosed chronic-phase chronic myeloid leukemia (CML-CP): subset analysis of the DASISION trial with 2-year follow-up. Int. J. Hematol. 99, 141-153 (2014).

4. Hughes, T. P. et al. Early molecular response predicts outcomes in patients with chronic myeloid leukemia in chronic phase treated with frontline nilotinib or imatinib. Blood 123, 1353-1360 (2014).

5. Marin, D. et al. Assessment of BCR-ABL1 transcript levels at 3 months is the only requirement for predicting outcome for patients with chronic myeloid leukemia treated with tyrosine kinase inhibitors. J. Clin. Oncol. 30, 232-238 (2012).

6. Hanfstein, B. et al. Early molecular and cytogenetic response is predictive for long-term progression-free and overall survival in chronic myeloid leukemia (CML). Leukemia 26, 2096-2102 (2012).

7. Branford, S. et al. Initial molecular response at 3 months may predict both response and event-free survival at 24 months in imatinib-resistant or -intolerant patients with Philadelphia chromosome-positive chronic myeloid leukemia in chronic phase treated with nilotinib. J. Clin. Oncol. 30, 4323-4329 (2012).

8. Baccarani, M. et al. European LeukemiaNet recommendations for the management of chronic myeloid leukemia: 2013. Blood 122, 872-884 (2013).

9. Lee, S. E. et al. Distinct predictive factors influence on achievement of early molecular response by frontline imatinib in chronic phase chronic myeloid leukemia. Leuk. Res. 39, 411-418 (2015).

10. Hanfstein, B. et al. Velocity of early BCR-ABL transcript elimination as an optimized predictor of outcome in chronic myeloid leukemia (CML) patients in chronic phase on treatment with imatinib. Leukemia 28, 1988-1992 (2014).

11. Branford, S. et al. Prognosis for patients with CML and 10\% BCR-ABL1 after 3 months of imatinib depends on the rate of BCR-ABL1 decline. Blood 124, 511-518 (2014).

12. Druker, B. J. et al. Five-year follow-up of patients receiving imatinib for chronic myeloid leukemia. N. Engl. J. Med. 355, 2408-2417 (2006). 
13. Hochhaus, A. et al. Six-year follow-up of patients receiving imatinib for the first-line treatment of chronic myeloid leukemia. Leukemia 23, 1054-1061 (2009).

14. Zhu, Y. \& Qian, S. X. Clinical efficacy and safety of imatinib in the management of $\mathrm{Ph}(+)$ chronic myeloid or acute lymphoblastic leukemia in Chinese patients. Onco. Targets Ther. 7, 395-404 (2014)

15. Li, Q. B. et al. Imatinib plasma trough concentration and its correlation with characteristics and response in Chinese CML patients. Acta Pharmacol. Sin. 31, 999-1004 (2010).

16. Qin, Y. Z. et al. Different kinetic patterns of BCR-ABL transcript levels in imatinib-treated chronic myeloid leukemia patients after achieving complete cytogenetic response. Int. J. Lab. Hematol. 30, 317-323 (2008).

17. Jain, P. et al. Early responses predict better outcomes in patients with newly diagnosed chronic myeloid leukemia: results with four tyrosine kinase inhibitor modalities. Blood 121, 4867-4874 (2013).

18. Iriyama, $\mathrm{N}$. et al. Shorter halving time of BCR-ABL1 transcripts is a nove predictor for achievement of molecular responses in newly diagnosed chronic-phase chronic myeloid leukemia treated with dasatinib: results of the D-first study of Kanto CML study group. Am. J. Hematol. 90, 282-287 (2015).
19. Fava, C. et al. Early BCR-ABL1 reduction is predictive of better event-free survival in patients with newly diagnosed chronic myeloid leukemia treated with any tyrosine kinase inhibitor. Clin. Lymphoma Myeloma Leuk. 16(Suppl), S96-S100 (2016).

20. El-Metnawy, W. H. et al. Predictive value of pretreatment BCR-ABL(IS) transcript level on response to imatinib therapy in Egyptian patients with chronic phase chronic myeloid leukemia (CPCML). Int. J. Biomed. Sci. 9, 48-53 (2013).

21. Huet, $\mathrm{S}$. et al. Major molecular response achievement in $\mathrm{CML}$ patients can be predicted by BCR-ABL1/ABL1 or BCR-ABL1/GUS ratio at an earlier time point of follow-up than currently recommended. PLOS ONE 9, e106250 (2014).

22. Branford, S. Monitoring and defining early response: where to draw the line? Best Pract. Res. Clin. Haematol. 29, 284-294 (2016).

23. Larson, R. A. et al. Imatinib pharmacokinetics and its correlation with response and safety in chronic-phase chronic myeloid leukemia: a subanalysis of the IRIS study. Blood 111, 4022-4028 (2008).

24. El Missiry, M. et al. Early BCR-ABL1 transcript decline after 1 month of tyrosine kinase inhibitor therapy as an indicator for treatment response in chronic myeloid leukemia. PLOS ONE 12, e0171041 (2017) 